\title{
FRECUENCIA DE SOLICITUD Y PREVALENCIA DE REACTIVIDAD DE LA PRUEBA NO TREPONÉMICA (VDRL) EN PACIENTES CON ABORTO EN EL HOSPITAL LOCAL DEL NORTE DE BUCARAMANGA, COLOMBIA
}

\section{Frequency of nontreponemal testing (VDRL) and prevalence of test reactivity in patients with miscarriage at Hospital Local del Norte in Bucaramanga, Collombia}

\author{
Ricardo Ortiz-Serrano, $\mathrm{MD}^{1}$; Víctor Mauricio Herrera-Galindo, $\mathrm{PhD}^{2}$; \\ Catalina Acuña-Pradilla, $M D^{3}$
}

Recibido: septiembre 23/13 - Aceptado: marzo 18/14

\section{RESUMEN}

Introducción: la adherencia a las recomendaciones para la tamización de sífilis en mujeres gestantes es fundamental en la prevención de lúes congénita. El objetivo de este estudio fue estimar la frecuencia de solicitud de prueba no treponémica y reactividad en mujeres que cursan con aborto.

Materiales y métodos: estudio de corte transversal basado en el registro de pacientes que atendieron a la consulta de urgencias de obstetricia del Hospital Local del Norte de Bucaramanga entre enero $1^{\circ} \mathrm{y}$ diciembre 31 de 2011, y que tuvieron un diagnóstico de aborto. En estas pacientes se determinó la prevalencia de solicitud de la prueba no treponémica (VDRL), así como de resultados reactivos.

Resultados: en el periodo de estudio se identificaron 233 mujeres con diagnóstico confirmado de

1 Especialista en Ginecología y Obstetricia. Profesor asociado, Universidad Autónoma de Bucaramanga. Bucaramanga, Colombia. rortiz@unab.edu.co

$2 \mathrm{PhD}$ en Epidemiología. Profesor titular, Universidad Autónoma de Bucaramanga. Bucaramanga, Colombia. vherrera@unab.edu.co

3 Candidata a especialista en Ginecología y Obstetricia, Universidad Industrial de Santander. Bucaramanga, Colombia. aborto. La edad promedio fue de 24,2 \pm 7,3 años, con una edad gestacional de 9,1 \pm 3,3 semanas. Se documentó evidencia de solicitud de prueba no treponémica en 135 pacientes (57,9\%; IC $95 \%$ : 51,6-64,3) de las cuales 5 (3,7 \%; IC $95 \%$ : 0,5-6,9) tuvieron resultados reactivos: 1 con títulos de 1:2; 3 con títulos de 1:4, y 1 con títulos de 1:32.

Conclusión: pese a las recomendaciones vigentes, la prevalencia de solicitud de prueba no treponémica en pacientes con aborto es baja. Lo anterior evidencia la necesidad de concientizar al personal de salud acerca de la importancia de cumplir a cabalidad el protocolo de atención a la gestante.

Palabras clave: aborto, sífilis, prueba no treponémica, solicitud.

\section{ABSTRACT}

Introduction: Adherence to the recommendations for syphilis screening in pregnant women is critical for congenital syphilis prevention. The objective of this study was to estimate the frequency of nontreponemal test orders and prevalence of test reactivity in women with miscarriage. 
Materials and methods: Cross-sectional study based on the registry of patients coming to the obstetrical emergency service at Hospital Local del Norte, Bucaramanga, between January $1^{\text {st }}$ and December 31 ${ }^{\text {st }}$, 2011, diagnosed with miscarriage. The prevalence of nontreponemal testing (VDRL) orders as well as of reactive results was assessed in these patients.

Results: During the study period, 233 women were identified to have a confirmed miscarriage. The mean age was $24.2 \pm 7.3$ and the mean gestational age was $9.1 \pm 3.3$ weeks. Evidence for nontreponemal test orders was documented in 135 patients (57.9\%; CI 95\%: 51.6-64.3); of them, 5 (3.7\%; CI $95 \%$ : 0.5-6.9) had reactive results: 1 with 1:2 titres; 3 with 1:4 titres and 1 with 1:32 titres.

Conclusion: Despite existing recommendations, the prevalence of nontreponemal test orders in patients with miscarriage is low. This points to the need to create awareness among the staff about the importance of following closely the protocol for the care of pregnant patients.

Key words: Miscarriage, syphilis, nontreponemal test, request.

\section{INTRODUCCIÓN}

La sífilis es una enfermedad de transmisión sexual producida por el Treponema pallidum, cuyo mecanismo de adquisición es principalmente por contacto sexual. Su presentación en mujeres en edad reproductiva puede tener efectos adversos, ya que si se presenta durante la gestación puede ocasionar complicaciones de diversa índole que van a depender del estadio clínico de la enfermedad, de la edad gestacional y del inicio temprano del tratamiento. Durante el embarazo, la sífilis primaria, secundaria y latente temprana genera una diseminación hematógena hacia el feto produciendo una reacción inflamatoria. Sin tratamiento, el $25 \%$ de las gestantes presentan abortos u óbitos fetales durante el segundo trimestre, $11 \%$ terminan en muerte fetal intrauterina hacia el final de la gestación, $13 \%$ en parto pretérmino o bajo peso al nacer, y hasta un
$20 \%$ de los recién nacidos tendrán manifestaciones clínicas de sífilis congénita (1).

A nivel mundial y en países desarrollados como Inglaterra, Francia, Alemania y Estados Unidos, la incidencia de sífilis ha aumentado durante los últimos diez años, especialmente en hombres que mantienen relaciones sexuales con parejas del mismo sexo (2). Según la Organización Panamericana de la Salud (OPS), en América Latina y el Caribe se estima que el número de gestantes por año con resultados positivos para sífilis que no reciben tratamiento durante el control prenatal es de aproximadamente 330.000 (3). Para el año 2002, la prevalencia de esta enfermedad durante la gestación en países latinoamericanos fue de 3,1\%, oscilando entre 1,0\% en Perú y 6,3\% en Paraguay (3). En 2003, de acuerdo con un estudio realizado en San Luis de Potosí, México, la prevalencia de sífilis gestacional fue de 0,3\% (4). En Bolivia, para el año 2004, la prevalencia de sífilis gestacional y congénita fue de 7,2 y 1,1\%, respectivamente (5). Por otra parte, en Haití, entre 2004 y 2006, la prevalencia reportada de sífilis gestacional fue de 7,6\% y la tasa de sífilis congénita fue de 7,7 por 1000 nacidos vivos (6).

En Colombia, la solicitud de la serología a toda mujer gestante es una práctica obligatoria según la Resolución 412, Guía de detección temprana de las alteraciones en el embarazo, del Ministerio de Salud y Protección Social (7). En una evaluación sobre prácticas clínicas en el cuidado de gestantes y recién nacidos realizada entre 2004 y 2005 en hospitales de Bogotá se encontró que la solicitud de la serología no se llevó a cabo en el 30,5\% de las embarazadas antes de la semana 20 de gestación y en el 34,0 \% después de la semana 20 (8); sin embargo, no se reportó la prevalencia de la infección. Según el Observatorio de Salud Pública de Santander, la razón de prevalencia de sífilis gestacional se mantuvo entre 3 y 4 por 1000 nacidos vivos entre los años 2009 y 2012, mientras que la incidencia de sífilis congénita osciló entre 0,9 y 1,1 casos por 1000 nacidos vivos para el mismo periodo (9), es decir, que están por encima de las metas fijadas por la OPS y la Organización 
Mundial de la Salud (OMS) (sífilis congénita por debajo de 0,5 por 1000 nacidos vivos) (10). Es poco lo que se conoce de la frecuencia en Colombia de infección por T. pallidum en mujeres con aborto.

El objetivo de este estudio fue determinar la prevalencia de solicitud y de reactividad de la prueba no treponémica (VDRL) en pacientes que presentaron aborto y que fueron atendidas en el Hospital Local del Norte de Bucaramanga (HLNB) durante el año 2011.

\section{MATERIALES Y MÉTODOS}

Estudio de corte transversal basado en el registro de la consulta de urgencias de Obstetricia, Sala de Partos, del HLNB, institución de primer y segundo nivel de complejidad que atiende pacientes del régimen subsidiado, correspondiente a estratos 1 , 2 y 3, a partir del cual se identificaron todas las pacientes con diagnóstico de aborto (aborto incompleto, aborto en curso, huevo anembrionado, aborto provocado, aborto séptico, aborto retenido) entre el $1^{\circ}$ de enero y el 31 de diciembre de 2011.

Sobre la base de la prevalencia de no solicitud de la serología reportada por Rojas et al. en embarazadas antes de la semana 20 de gestación (30,5\%) (8), y considerando una casuística de aproximadamente 400 consultas anuales conducentes al diagnóstico de aborto o amenaza de aborto, se estimó que el estudio alcanzaría una precisión $\pm 5 \%$. Se analizaron todos los casos consecutivos durante el periodo determinado para la investigación.

Se identificaron todas las pacientes con diagnóstico de aborto o amenaza de aborto registradas en el libro de consultas de urgencias de Obstetricia, Sala de Partos, del HLNB. Posteriormente, en el archivo de la institución, se realizó la búsqueda manual de las historias clínicas correspondientes a las pacientes identificadas, excluyendo aquellas gestantes con amenaza de aborto en las que hubo evidencia de continuación del embarazo por encima de las 20 semanas y aquellas en las que no se pudo determinar la continuación del embarazo o la confirmación de aborto. Los autores emplearon un formato de recolección de datos para la extracción de la información correspondiente a la fecha de la primera consulta, edad materna, fórmula obstétrica, edad gestacional, registro de solicitud y resultado de la prueba no trepónemica, así como el tratamiento prescrito. En caso de no hallar evidencia de solicitud o reporte del resultado de la serología en la historia clínica, se consultó la base de datos del laboratorio clínico del HLNB para completar dicha información.

Se evaluaron las siguientes variables: edad, edad gestacional, evidencia de solicitud de la prueba serológica para sífilis y títulos de dilución de la prueba no treponemica (VDRL).

Todos los datos fueron digitados en una base de datos electrónica (Excel ${ }^{\circledR}$, Microsoft Office) diseñada para tal fin. Se realizó la descripción estadística de los datos empleando medias y prevalencias con sus respectivos intervalos de confianza del $95 \%$ para las variables continuas y discretas, respectivamente. La preparación y el análisis de los datos se realizó en el paquete estadístico Stata MP/12.1.

El estudio contó con la aprobación del Comité de Ética en Investigaciones de la Universidad Autónoma de Bucaramanga (UNAB).

\section{RESULTADOS}

Se identificaron 409 registros de pacientes que consultaron por amenaza de aborto o aborto durante el periodo de estudio. De estos se confirmó el diagnóstico de aborto en 233 (figura 1).

Entre las 233 pacientes que presentaron aborto se encontró una edad media de 24,2 años $\pm 7,3$ años, y una edad gestacional media de 9,1 semanas $\pm 3,3$ semanas. Tanto la solicitud como el reporte se documentaron en 135 mujeres (57,9\%; IC $95 \%$ : 51,6-64,3). En 5 pacientes de este último grupo (3,7\%; IC $95 \%$ : 0,5-6,9) la serología fue reactiva, así: 1 con títulos de 1:2; 3 con títulos de 1:4 y 1 con títulos de 1:32; sin embargo, en ninguno de estos casos se realizó una prueba confirmatoria. Todas las pacientes con serología reactiva recibieron tratamiento con penicilina benzatínica (tres dosis de 2.400.000 UI IM). 
Figura 1.

Flujograma

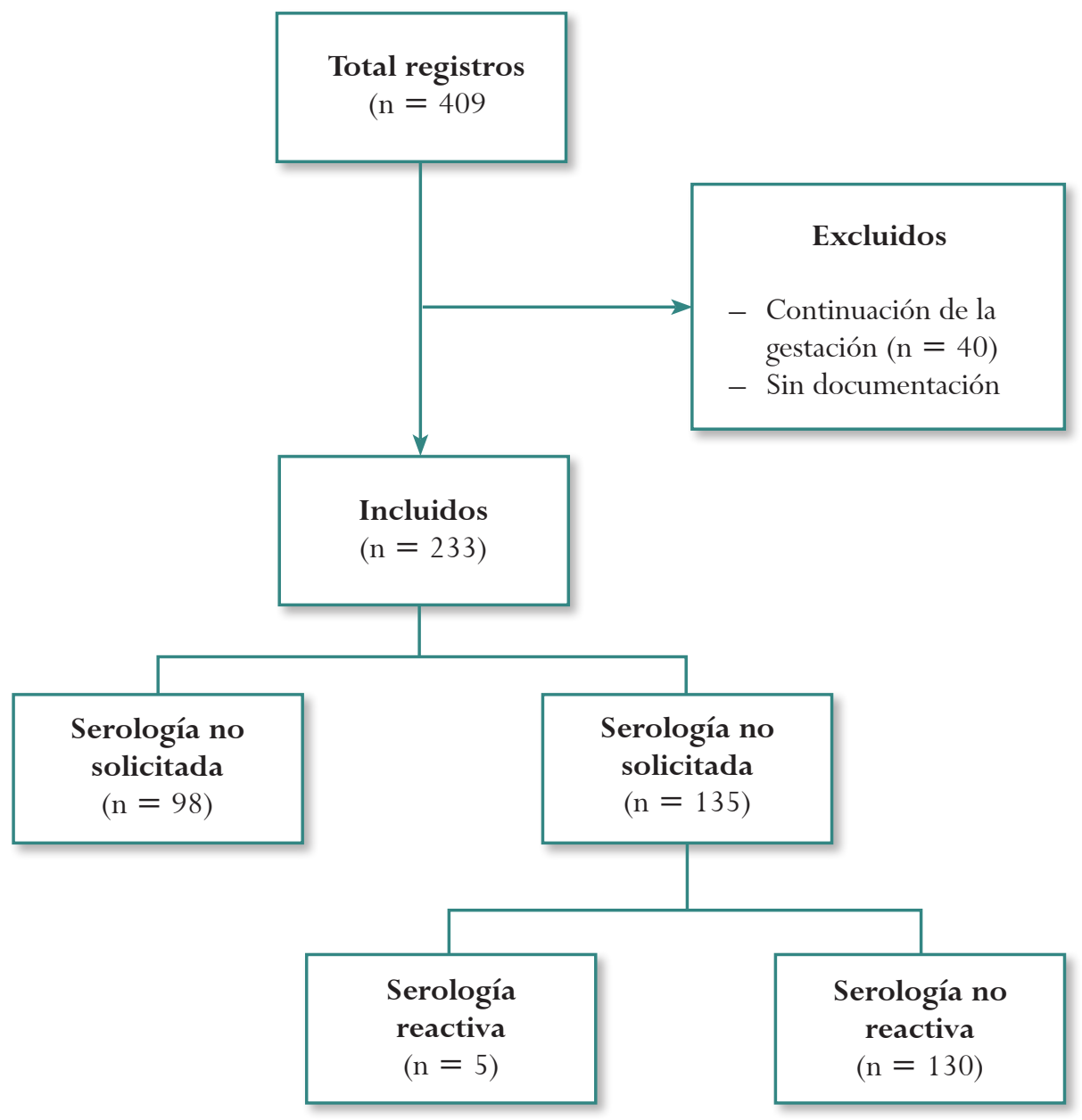

\section{DISCUSIÓN}

Este es un estudio descriptivo de corte transversal en el cual se evaluaron las historias clínicas de pacientes que consultaron al servicio de urgencias de Obstetricia, Sala de Partos, del HLNB con diagnóstico de aborto. Se encontró que la prevalencia de solicitud de prueba no treponémica (VDRL) en dichas pacientes fue de 57,9\%, mientras que la prevalencia de pruebas reactivas fue del 3,7\%.

Dado que la normatividad emanada del Ministerio de Salud y Protección Social obliga a la solicitud universal del VDRL a toda paciente embarazada en el primer control prenatal con el fin de realizar tamización para sífilis, sorprende encontrar una cifra de solicitud de esta prueba tan baja en este estudio, considerando que los médicos responsables del servicio en mención conocen a cabalidad los protocolos de atención a este respecto. Al comparar este resultado con lo encontrado en Bogotá por Rojas et al. (8) entre 2004 y 2005, se aprecia que la solicitud de VDRL antes de la semana 20 de gestación fue de 69,5\%, y en el presente estudio que fue de $57,9 \%$, lo cual permite concluir que a pesar de conocerse de antemano los algoritmos de manejo se deja pasar por alto la solicitud de un examen sencillo, económico y que nos permite sospechar una enfermedad que sigue manteniendo en niveles subóptimos uno de los indicadores de calidad en la 
atención en salud en nuestro país. Es probable que al tratarse de pacientes con aborto se genere una falsa sensación de relajación en cuanto a la exigencia de cumplir con los protocolos establecidos para atención de gestantes, lo cual podría explicar la diferencia de solicitud del examen encontrada con el estudio de Rojas et al. (8).

A las pacientes objeto de este estudio no se les realizó prueba treponémica confirmatoria debido a que para la fecha en que se realizó la recolección de la muestra la contratación del examen de Fluorescent Treponemal Antibody Absorption (FTA-ABS) (Inmunofluorescencia indirecta con absorción del suero) se había realizado con una entidad estatal, cuyos resultados presentaban demoras que convertían el test en una prueba poco útil. Por esta razón, todas las pacientes con serologías reactivas fueron tratadas, quizás, sobrestimando la presencia de la enfermedad. Esta limitación no permitió el cálculo de la prevalencia real de sífilis en la población estudiada.

Además, de acuerdo con el protocolo de atención para esta patología, recomendado por el Ministerio de Salud y Protección Social, las pacientes que presenten serologías mayores a 1:8 se deben tratar sin la necesidad de solicitar pruebas confirmatorias. Este protocolo va en contravía con las guías de atención emanadas del CDC que recomienda realizar pruebas treponémicas confirmatorias sin importar las diluciones de la serología (11).

Este estudio es el primero que se realiza en la ciudad, específicamente para evaluar a mujeres con aborto, con un número significativo de casos, lo que sugiere que a futuro pueda determinarse la prevalencia de sífilis en pacientes con aborto espontáneo.

De las 409 mujeres inicialmente encontradas en las diferentes búsquedas realizadas, en 136 no se supo si el embarazo continuó debido a que el registro inicialmente encontrado reportaba una amenaza de aborto y no existen más anotaciones posteriores en la historia clínica de estas pacientes. Este factor pudo haber llevado a una subestimación de la frecuencia del resultado positivo.
Se considera necesario concientizar a los médicos tratantes de los servicios de urgencias sobre la necesidad de realizar esta prueba como parte integral del manejo de la paciente con aborto. Estos hallazgos permiten recomendar la solicitud rutinaria de la serología a todas las pacientes con aborto, considerando que la solicitud de esta prueba está contemplada dentro del protocolo de atención de la mujer gestante recomendado por el Ministerio de Salud y Protección Social en nuestro país (7).

Financiación: el presente estudio fue financiado con fondos de la Convocatoria Interna 2010-2012 de la Universidad Autónoma de Bucaramanga (UNAB).

\section{REFERENCIAS}

1. Schmid GP, Stoner BP, Hawkes S, Broutet N. The need and plan for global elimination of congenital syphilis. Sex Transm Dis. 2007;34:S5-10.

2. Doherty L, Fenton KA, Jones J, Paine TC, Higgins SP, Williams D, et al. Syphilis: old problem, new strategy. BMJ. 2002;325:153-6.

3. Organización Panamericana de la Salud, Unidad VIH/SIDA. Hoja informativa sobre sífilis congénita. Washington, D.C; 2004. [Visitado 2014 Feb 11]. Disponible en: http://www.paho.org/Spanish/AD/ FCH/AI/EliminaSifilisLAC.pdf

4. Noyola DE, Malacara-Alfaro O, Lima-Rogel V, TorresMontes A. Seroprevalence of syphilis in pregnant women in San Luis Potosí. Salud Pública Mex. 2006;48:151-4.

5. Revollo R, Tinajeros F, Hilari C, García SG, Zegarra L, Díaz-Olavarrieta C, et al. Maternal and congenital syphilis in four provinces in Bolivia. Salud Pública Mex. 2007;49:422-8.

6. Lomotey CJ, Lewis J, Gebrian B, Bourdeau R, Dieckhaus K, Salazar JC. Maternal and congenital syphilis in rural Haiti. Rev Panam Salud Pública. 2009;26:197-202.

7. Ministerio de Protección Social. Guías de promoción de la salud y prevención de enfermedades en la salud pública. Bogotá, Colombia, mayo 2007. [Visitado 2014 
Feb 11]. Disponible en: http://www.minsalud.gov.co/ Documentos\%20y\%20Publicaciones/GUIA\%20 DE\%20ATENCION\%20DE\%20LA\%20SIFILIS\%20 CONGENITA.pdf.

8. Rojas-Higuera R, Londoño-Cardona JG, ArangoGómez F. Clinical practice in looking after breastfeeding women and the new-born in some hospitals in Bogotá, Colombia. Rev Salud Pública (Bogotá). 2006;8:223-34.

9. Ochoa M. Sífilis gestacional y congénita. Santander, 2009-2012. Informe Epidemiológico de Santander. 2012;6(3).
10. Valderrama, J. Eliminación de sífilis congénita en América Latina y el Caribe: Marco de referencia para su implementación. Washington, D.C: OPS, 2005. [Visitado 2014 Feb 11]. Disponible en: http://www1. paho.org/Spanish/AD/FCH/AI/EliminaSifilisLAC.pdf. 11. Workowski KA, Berman S. Centers for Disease Control and Prevention (CDC). Sexually Transmitted Diseases Treatment Guidelines. MMWR Recomm Rep. 2010;59:1-110. 